\title{
X. Über die Überlieferung der Urkunden
}

Nach den Gepflogenheiten der modernen Kritik scheint es selbstverständlich, daß, wenn ein Herausgeber in der Abschrift eines Actenstückes, das er wiedergeben will, Fehler findet und die Originalurkunde oder eine besser erhaltene Abschrift in einer andern Sammlung zur Verfügung bat, er diese verschiedenen Texte gegeneinanderhält und aus ihrer Vergleichung für die Verbesserung seiner Abschrift Nutzen zieht. Unter dem Druck dieser vorgefaßten Meinung habe ich den Text der Urkunden Theodorets untersucht und aufgestellt. Nach wiederbolter Prüfung habe ich mich jedoch dem klaren Tatbestand beugen müssen, der mir mehr und mehr die sichere Erkenntnis aufzwang: während es eine Fülle von Beweisen gegenseitiger Beeinflussungen der Handschriften bei Theodoret selber gibt, findet sich in den von ihm aufgenommenen Urkunden nicht die geringste Spur einer Vergleichung mit dẹn andern Quellen weder als einer methodisch durchgeführten noch in Form vereinzelter und sporadischer Besserungen. So hat für mich schließlich die Frage ein geradezu umgekehrtes Aussehen gewonnen, wie ich lange Zeit angenommen hatte. A priori und ganz allgemein müssen die Urkunden Theodorets für unberührt von jeder Nachvergleichung gelten, die der Überlieferung des Autors selber fremd ist. Wer davon in besondern Fällen Spuren zu entdecken glaubt, hat dafür den Beweis zu liefern.

Praktisch wäre es in der Tat überflüssig und Zeitverschwendung, hier zur Beweisführung Beispiele anzuhäufen, die immer zu demselben negativen Schluß führen würden: die und die richtige Lesart von A oder $B$ allein darf nicht aufgefaßt werden als wiedereingeführt im Anschluß an Athanasius, Socrates oder eine beliebige andre Quelle, von der sie bestätigt wird. Sie erklärt sich vollkommen durch den allgemeinen Charakter der Texterhaltung in der ganzen Handschrift. Übrigens findet man in derselben Handschrift neben dieser richtigen Lesart offenbaren Unsinn oder Auslassungen, die bei der Nachvergleichung auf Grund der Quellen zweifellos verschwunden sein würden. Denn eine nach einem andern Autor vorgenommene Collationierung muß immer etwas methodisch verfahren und verrät sich durch eine Reihe aufeinanderfolgender Beispiele - ganz so wie sich die so häufige gegenseitige Beeinflussung der Handschriften desselben Autors leicht erkennen läßt. Die gleichen Handschriften also, die in den erzählenden Abschnitten Vertrauen verdienen, tun es auch in den Urkunden, und jede bleibt in ibrem Verhalten durchweg sich selber gleich. Mögen die Überarbeiter tendenziös wie $\mathrm{T}$ oder geschickt wie $\mathrm{H}$ sein, sie machen von ihrer Veranlagung bei den Urkunden denselben Ge- 
brauch wie in der Erzählung; ihre Änderungen sind immer der Ausfluß einer freien Combination, niemals eines Zurückgreifens auf die Quellen.

Das Fehlen dieser Art von Überarbeitung, die für unsere Handschriften bezieugt ist, läßt sich nun gerade so für Theodoret selber dartun. Bei ihm erklärt es sich hauptsächlich durch eine Arbeitsmethode, die wir von Theodoret bis Nicephorus, d. h. vom Anfang bis zum Ende der byzantinischen Periode, feststellen können. Die Urkunden wurden einfach nach einem dem Schreiber bezeichneten Text zum Copieren gegeben, und der Autor nahm sich allem Anschein nach oft nicht die Mühe, die Abschrift desselben wieder durchzusehen. In dieser Hinsicht habe ich oben S. XLIII $f$ an charakteristischen Beispielen gezeigt, wie Nicephorus verfahren ist.

$\mathrm{Da}$ Theodorus Lector, der auch dauernd die drei Synoptiker zur Hand hat, jemals in den Urkunden ihre Texte ineinander gearbeitet hätte, davon habe ich kein sicheres Anzeichen entdeckt.

Cassiodor hält bei seinen Übersetzungen von Actenstücken treu daran fest, die Recension des von ihm gewählten Autors lateinisch wiederzugeben. Wenn diese Recension verderbt oder unverständlich ist, verliert er den Faden, setzt neu an oder gibt auch alle Hoffnung auf und läßt das Unverständliche einfach aus. Niemals kommt er auf den Gedanken, daß z. B. Socrates, dem er den unmittelbar folgenden Abschnitt entnimmt, ihm den Schlüssel zu der Stelle geben könnte, die ihm im Augenblick bei Theodoret Not macht. Noch viel weniger läßt er sich bei den aus dem Lateinischen ins Griechische übertragenen Urkunden auch nur einfallen, was in der Gegenwart jeder tun würde, sich der mühsamen Arbeit einer Rückübersetzung zu entziehen und den Text des lateinischen Originals zu ermitteln, das wiederaufzufinden ihm zuweilen wohl ein leichtes gewesen wäre.

Bei Cassiodor sehe ich nur einen bemerkenswerten Fall, der etwas ungewöhnlich scheint. Die bei ihm II 12 wiedergegebene Urkunde ist nicht nach Theodoret 19 übersetzt, wie die Handschriften und Ausgaben behaupten, sondern nach Socrates I 9; sie ist übrigens zwischen andere Excerpte aus Socrates eingeschoben. Für die Schlußformel (S. 41, 20 ff) indessen stimmt Cassiodor mit Theodoret und nicht mit Socrates überein. $\mathrm{Zu}$ beachten ist, daß Gelasius und die Handschriften des Athanasius, die eine unabbängige Überlieferung derselben Urkunde darstellen, eine weitere abweichende Schlußformel haben.

Dieser Fall bei Cassiodor kann zu verschiedenen Hypothesen Anlaß geben, aber unter keinen Umständen würde ich. die Erklärung dafür in einer beabsichtigten Beeinflussung durch den Text des Theodoret bei diesen letzten Zeilen suchen. Ich möchte vielmehr annehmen, daß diese 
Schlußvarianten auf die ursprüngliche Urkunde zurückgehen und daß Cassiodor in seiner Handschrift des Socrates beide Formeln gefunden hatte.

Gelasius Cyzicenus nimmt mit seinem völligen Mangel an Respekt vor dem Buchstaben der Urkunden, die er wiedergibt, eine Sonderstellung ein. Sicher hat er sie nicht einfach copieren lassen, sondern er hat sie dictiert oder selber geschrieben, indem er sie öfters interpolierte, fälschte und überarbeitete. Aber diese Änderungen entspringen seiner freien Erfindung, ohne eine Spur von Mischung mehrerer Quellen, so daß es trotz aller seiner Freiheiten möglich bleibt zu bestimmen, welcher Autor für ihn den Ausgangspunkt bildete.

Die Urkunden bei Theodoret bieten zahlreiche Gelegenheiten, die vorstehenden Thesen zu belegen. So hahen wir für den Brief des Eusebius von Cäsarea:

1) Vier Copien, die, unabhängig voneinander, derselben Sammlung entnommen sind, nämlich bei Theodoret I 12, in den Handschriften des Athanasius ( $\mathrm{PG}$ 20, 1536), bei Gelasius Cyzicenus II $34 \mathrm{f}$ und bei Socrates I 8. Ein Blick auf den kritischen Apparat genügt, um zu zeigen, daß Gelasius nicht Theodoret abschreibt, wie man geglaubt hat.

2) Eine nach Theodoret gefertigte Copie bei Nicephorus VIII 22. Nicephorus läßt sogar am Schluß die sonderbare Lesart 54, 2 ż $\mu \varphi \alpha \nu \tilde{\eta}$

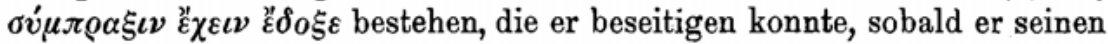
Socrates zu Rate zog.

3) Eine Copie von Theodorus Lector und eine Utbersetzung (welche die beiden Symbole ausläßt) bei Cassiodor II 11, beide auf Socrates beruhend. Allerdings würde die Tatsache, daß diese beiden Versionen durchaus von Socrates abhängig sind, aus einer Vergleichung mit dem bisher bekannten Texte des Socrates nicht mit voller Deutlichkeit hervorgehn. Gerade der herkömmliche Socrates-Text, der auf FM beruht, ist häufig entstellt. Die Benutzung der Handschrift A des Socrates, von T und von Cassiodor gestattet in einer großen Zahl von Fällen einen mit der Überlieferung der andern Autoren gleichlautenden Text wiederherzustellen. Ich habe mich hiervon durch eine Untersuchung aller Varianten des Briefes überzeugt, die ich an dieser Stelle wiederzugeben nur darum nicht für angezeigt halte, weil sie speciell die Überlieferung des Socrates angeht. Auf keinen Fall haben Theodorus und Cassiodor, deren Überlieferung voneinander getrennt verläuft, sich begegnen können, um im Anschluß an eine andre Quelle in ihren Text eine Menge identischer, oft belangloser Änderungen (z. B. hinsichtlich der Wortstellung) einzuführen, die bei Socrates gerade mit der gemeinen Überlieferung der Urkunde zusammentreffen. Anderseits bewahren beide an andern Stellen desselben Actenstücks die besondern Züge der echten socratischen Überlieferung und 
bemerken nicht einmal die Läcke von ftinf bis sechs Zeilen (Hussey S. 56, 7), die sich in dieser Überlieferung befand.

Vergleichendes Studium führt bei den andern Urkunden zu ähnlichen Resultaten; z. B. Theodoret I 9, wo die Überlieferung auch sehr reich ist. Hier ist die theodoretische Version besonders fehlerhaft. Kein Schreiber hat daran gedacht, auf Socrates zurückzugreifen, um sie zu verbessern und offenbare Auslassungen wie z. B. das Wort $\tau \iota \mu \eta \dot{\nu}$ 40, $7 \mathrm{zu}$ ergänzen.

Die mechanische Art, in der von Anfang an die Urkunden copiert worden sind, scheint sich noch durch andre Anzeichen zu verraten.

Theodoret reproduciert I 15 u. 16 zwei Briefe Constantins, die von Socrates I 9, $47 \mathrm{ff}$ nicht bloß in derselben Ordnung, sondern auch mit einem identischen Erzählungssatz als Übergang (Theodoret S. 59, 20-24= Socr. I 9,50$)$ gebracht werden.

Auf den ersten Blick glaubt man da einen sichern Beweis der Tatsache zu haben, daß Theodoret von Socrates direct abschreibt. Bei genauerer Prüfung stellt sich die Frage als weit weniger einfach heraus. Sicher steht Theodorets Text Socrates näher als Eusebius, der gleichfalls diese Actenstücke wiedergibt. Dennoch hat Socrates manche Besonderheiten, bei denen man nicht verstehen würde, daß Theodoret sie vermeidet, wenn er direct von Socrates abgeleitet wäre. Man müßte in diesem Falle annehmen, daß unsere ganze Überlieferung des Socrates anf eine hinterdrein entstellte Quelle zurückgehe; diese Hypothese ist nun aber für einen Fall wie 60, 6: $\delta \varepsilon \delta \delta \xi \xi$ Theodoret $=$ Gel.Eus. $>$ Socr. Codd.Cass. kaum zulässig, was daran denken läßt, daß Cassiodor für dieses Actenstück unseren Text des Socrates benutzt hat.

$\mathrm{Da}$ Theodoret sich im allgemeinen wohl hütet, von einem seiner Vorgänger wörtlich abzuschreiben, da er außerdem genug andre Quellen zur Verfügung hat, um sich dessen enthalten zu können, bin ich geneigt anzunehmen, daß er diese beiden Actenstücke derselben Sammlung entnommen hat, aus der sie auch Socrates schöpfte, und daß beide dort einen identischen Übergang vorgefunden haben, der von ihren Schreibern gleichzeitig mit den Briefen copiert worden ist. Es handelt sich wohl um eine Sammlung von Briefen Constantins, auf die Socrates (I 9,6466, vgl. I 38, 4) gerade in eben diesem Abschnitt seines Werkes anspielt. Socrates spricht von jenen Briefen als von Texten, die sich selbst zu verschaffen jedem seiner Leser leicht falle. Sie waren wohl schon mit anderen Urkunden zu größeren Sammlungen vereinigt. Solche Sammlungen enthielten nun aber neben den Urkunden sicher einen erklärenden Teil (vgl. Ed. Schwartz, Zur Geschichte des Athanasius VI, in den Nachrichten der kgl. Ges. der Wiss. zu Göttingen, 
phil.-hist. Klasse 1905, S. 259). Socrates und Theodoret haben in derselben Sammlung den Brief an Macarius (Socr. I 9, 56; Theod. I 17) gelesen und ebenso den Brief Constantins gegen Eusebius von Nicomedien, der für diese Frage nach der Herkunft sehr bezeichnend ist; denn Theodoret reproduciert ihn (I 20), Socrates bringt einfach eine Inhaltsangabe von ihm $(I 9,65)$ und Eusebius erwäbnt ihn begreiflicherweise gar nicht.

Ich bin geneigt zu glamben, daß wir dieser Methode mechanischen Copierens bei Theodoret II 8 die Erhaltung des wertvollen Symbolums von Sardica verdanken. Für das ganze Capitel II 8 schöpft Theodoret ebenso wie die lateinische Übersetzung des Codex Veronensis aus einer Conciliensammlung, und da ein solches Corpus auch erzählende Teile enthielt, ist es wohl dieselbe Quelle, die Theodoret am Anfang von II 7 mit $\tau \dot{\alpha} \pi \alpha \lambda \alpha i \dot{\alpha}$ $\delta \iota \eta \gamma \eta \dot{\eta} \mu \tau \alpha$ bezeichnet. Athanasius hat die Gültigkeit des Symbolum Sardicense bestritten (cf. ad Antioch. 5) und sich wohl gehütet, obgleich er wahrscheinlich aus derselben Sammlung schöpft wie Theodoret, es am Ende des Synodalbriefes als authentisches Actenstück wiederzugeben. Hilarius hat gleichfalls dieses Glaubensbekenntnis ignoriert. Wenn Theodoret, für den Athanasius die hohe theologische Autorität ist, dennoch das verdächtige Actenstück wiedergibt, so hat er sich wohl nicht die Mühe genommen, über die Tragweite der Urkunde nachzudenken, die er abschreiben ließ; ja vielleicht hat er sie überhaupt nicht wieder durchgelesen, andernfalls wturde er nicht eine Copie haben abschreiben lassen, deren Text an so vielen Stellen kaum erständlich ist.

Man kann sich auch fragen, ob das allgemeine Urteil, welches S.118 5-9 auf die Urkunde folgt, wirklich von Theodoret selber stammt. In den Handschriften sind diese Worte direct an das Sardicense angefügt, und das neue Capitel beginnt (außer in A) unmittelbar nach ihnen mit den Worten: Taṽ $\boldsymbol{\alpha} \alpha$ ó Kóv $\sigma \tau \alpha \varsigma(118,10)$. Gleichfalls unmittelbar vor diesen letzteren Worten schließt Cassiodor sein Capitel und verläßt die Tripartita den Theodoret, um Sozomenus zu folgen. Endlich hören in A die Anführungsstriche, die jede Zeile der Urkunde einfassen, erst bei unserer Zeile 118, 9 auf. Ich bin daher geneigt zu glauben, daß der Schreiber das ihm angegebene Capitel ganz und gar copiert hat und daß die Zeilen 118, 5-9 eine Betrachtung sind, die mit der Urkunde aus der Quelle selber in Theodoret übergegangen ist.

Um noch ein weiteres Beispiel herauszugreifen, so scheint es in Capitel IV, 8 wirklich, daß Theodoret nur die Aufschrift ( $x \alpha \grave{i}$ Ovंá $\lambda \eta s$ $220,6)$ des Briefes gelesen hat; sonst würde er nicht den Schluls ausgesprochen oder wiedergegeben haben, daß Valens zu dieser Zeit rechtgläubig war. Wenn er diese sonderbare Urkunde auch nur mit 
ein wenig Aufmerksamkeit gelesen hätte, würde er jedenfalls trotz des schlechten Textzustandes bemerkt haben, daß Valentinian sich darin gerade über die Ketzerei und die Verfolgungen des Valens beklagt.

Durch die vorstehenden Betrachtungen scheint mir die Annahme beseitigt zu sein, daß die Handschriften Theodorets nach den Parallelautoren oder nach den Quellen corrigiert wären. Ihre Schreiber haben an ein Verfahren, das uns heute so selbstrerständlich scheint, gar nicht gedacht. Freilich werde ich mich wohl hüten zu behaupten, daß das Studium der Überlieferung anderer Autoren immer zu einem ähnlichen Schluß führen würde. De Boor ist zwar für Theophanes (Theophanis Chronographia II 436 ff) gleichfalls zu diesem Schluß gekommen; aber ich sehe z. B., daß Ed. Schwartz (Eusebius Kirchengeschichte III, S. CLXIV ff) für die Auszüge des Josephus festgestellt hat, daß manche Handschriften des Eusebius nach der Quelle corrigiert sind (s. auch de Boor, Georgii Monachi Chronicon LVIf). Es ist übrigens begreiflich, daß an eine solche Nachvergleichung leichter bei wohlbekannten und klar bezeichneten Autoren gedacht wird als bei Urkunden. Ebenso müssen die Citate aus der Bibel besonders beurteilt werden, und das Verfahren der Chronisten, die sich fortwährend der Reihe nach ergänzen, indem sie sich auf dieselben Quellen beziehen, stellt noch einen weitern, wieder ganz verschiedenen Arbeitsmodus dar.

\section{Die Abschreiber}

Das Verzeichnis der Abschreiber, das sich S. $366 \mathrm{f}$ dieser Ausgabe findet, beabsichtigt keineswegs, alle Autoren aufzuzählen, die aus der Kirchengeschichte Stellen entlehnt haben. Ich habe mir besonders angelegen sein lassen, die Autoren heranzuziehen, für welche zuverlässige kritische Ausgaben vorliegen, z. B. Georgius Monachus und Theophanes von de Boor sowie das Synaxarium ecclesiae Constantinopolitanae von Delehaye.

Unter den Anleihen aus Theodoret beanspruchen einen Platz für sich die Excerpte aus der Tripartita des Theodorus Lector, z. B. in Theophanes, Julius Polydeukes, den Anecdota Paris. von Cramer Bd. II, S. $87 \mathrm{ff}$, dem Baroccianus 142, Bl. $216 \mathrm{ff}$ (ich habe für diese Excerpta Barocciana die Abschrift de Boors zu Rate ziehen können) und anderwärts. Im allgemeinen hat die Durchforschung dieser zahlreichen Bruchstücke kaum neues Licht über den Zustand des Textes gebracht, und ich habe in Verweisungen zurückhaltend sein können. Die Frage der Epitome (s. darüber Bidez, La tradition manuscrite de Sozomène, S. $44 \mathrm{f}$ und $77 \mathrm{ff}$ ) würde eine besondere Untersuchung 